\title{
Simulation of EMIC wave excitation in a model magnetosphere including structured high-density plumes
}

\author{
Lunjin Chen, ${ }^{1}$ Richard M. Thorne, ${ }^{1}$ and Richard B. Horne ${ }^{2}$ \\ Received 3 March 2009; revised 20 April 2009; accepted 15 May 2009; published 23 July 2009.
}

[1] The HOTRAY code is used to evaluate the path integrated gain of electromagnetic ion cyclotron (EMIC) waves as a function of frequency in two propagation bands above the $\mathrm{O}^{+}$and $\mathrm{He}^{+}$gyrofrequencies. Calculations are performed over a range of $\mathrm{L}$ shell $(3<L<7)$ assuming a cold $\mathrm{H}^{+}-\mathrm{He}^{+}-\mathrm{O}^{+}$plasma with an additional bi-Maxwellian hot ring current proton distribution. The cold plasma model includes a plasmasphere and high-density storm time plume region containing spatial density fluctuations.

The strongest wave gain $(>40 \mathrm{~dB})$ is found near the plasmapause, within regions with density structure in the plume, and in the low-density trough at $L \geq 6.5$. As a selfconsistent test on whether EMIC waves play an important role in relativistic electron loss from the radiation belts, the minimum cyclotron resonant electron energy is evaluated as a function of wave frequency and L shell for those EMIC waves that exhibit significant gain. The lowest electron resonant energies (approximately a few $\mathrm{MeV}$ ) are found in structured plumes. The sensitivities of both the wave gain and electron minimum resonant energy to variation in thermal ion compositions, the energetic proton properties, or plume density structure are also investigated.

Citation: Chen, L., R. M. Thorne, and R. B. Horne (2009), Simulation of EMIC wave excitation in a model magnetosphere including structured high-density plumes, J. Geophys. Res., 114, A07221, doi:10.1029/2009JA014204.

\section{Introduction}

[2] Electromagnetic ion cyclotron (EMIC) waves can be excited by an anisotropic distribution of energetic ring current ions, with energies of a few tens keV. Previous studies [Gomberoff and Neira, 1983; Young et al., 1981; Horne and Thorne, 1993] have shown that the generation and propagation characteristics of EMIC waves in a multiion $\left(\mathrm{H}^{+} ; \mathrm{He}^{+} ; \mathrm{O}^{+}\right)$plasma are profoundly controlled by ion fractional composition and by the ratio $\omega_{p e} /\left|\Omega_{e}\right|$, i.e., the ratio of electron plasma to gyrofrequency. EMIC waves can also be guided by density gradients at the plasmapause, which keep the wave normal angle small and significantly enhance the path integrated gain [Thorne and Horne, 1997].

[3] EMIC waves excited during storms can also resonate with relativistic electrons, causing rapid scattering loss [Thorne and Kennel, 1971], but the minimum energy for electron resonance is strongly dependent on ion composition and plasma density [Summers and Thorne, 2003; Albert, 2003; Li et al., 2007]. A statistical study of EMIC waves observed on CRRES [Meredith et al., 2003] showed that the minimum resonant energy for electrons was gener-

\footnotetext{
${ }^{1}$ Department of Atmospheric and Oceanic Sciences, University of California, Los Angeles, California, USA.

${ }^{2}$ British Antarctic Survey, Natural Environment Research Council, Cambridge, UK.
}

Copyright 2009 by the American Geophysical Union. 0148-0227/09/2009JA014204 ally $\geq 2 \mathrm{MeV}$ for the majority of EMIC wave events, but about $11 \%$ of reported EMIC events had minimum resonant energy $<2 \mathrm{MeV}$. EMIC waves with amplitude up to $10 \mathrm{nT}$ have been observed during the main phase of magnetic storm [Erlandson and Ukhorskiy, 2001], capable of inducing strong scattering of relativistic electrons at a rate comparable to the strong diffusion limit [Shprits et al., 2009]. Such loss is confined to high-density regions just inside the plasmapause or within dayside drainage plumes [Thorne et al., 2006; Jordanova et al., 2008]. Electron density inside such storm time plumes exhibits fine-scale structures [Goldstein et al., 2004; Spasojević et al., 2003, 2004]. Furthermore, as illustrated in Figure 1, such plumes tend to be wrapped around the plasmapause during the storm recovery, leading to radial density gradients, which can strongly influence EMIC wave propagation, in afternoon dust MLT sectors. Such density structure in the plume has not been included in previous simulations of wave excitation. Consequently, in this study, detailed ray tracing is performed with the HOTRAY code [Horne, 1989] to examine the most favorable regions for EMIC wave excitation, the typical frequency distribution of excited waves, and the ability of the waves to scatter energetic electrons.

[4] The outline of this paper is as follows. In section 2, a model of thermal plasma density is described. Ray tracing results together with calculation of minimum electron resonant energy are presented in section 3, and the sensitivity of simulated EMIC wave gains and corresponding minimum electron resonant energy to thermal ion compo- 


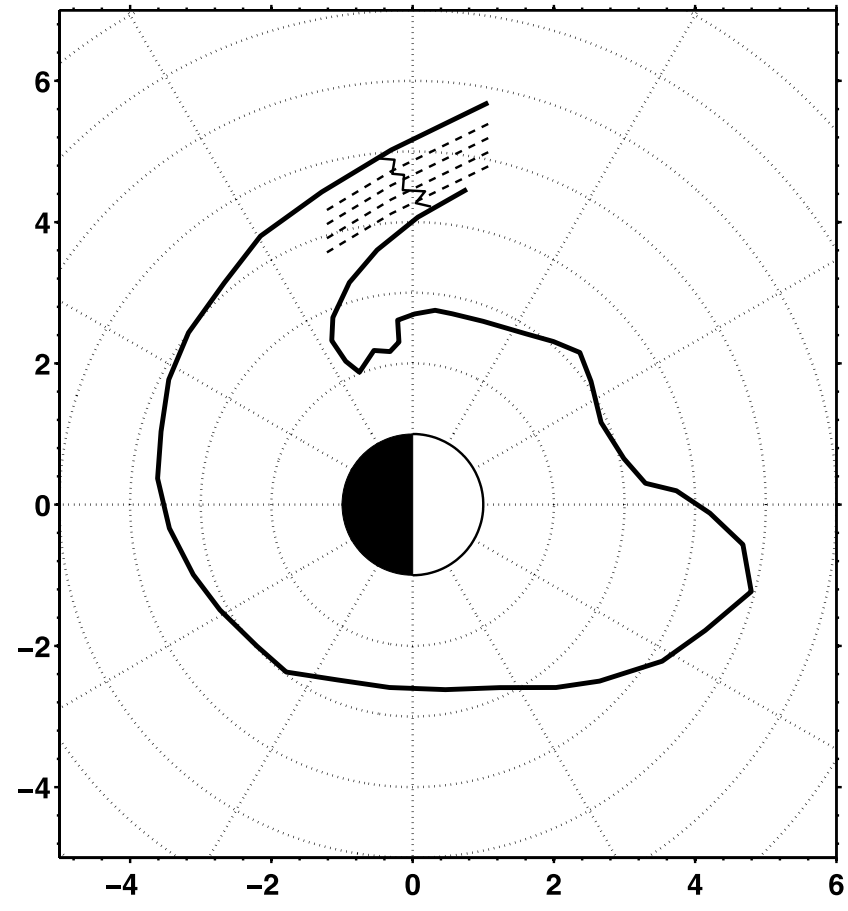

Figure 1. Schematic diagram of the plasmapause location and attached plume structure wrapping around during storm time condition, reproduced from EUV measurement at 0939 UT 10 June 2001 [Spasojević et al., 2003, Figure 12c]. Wiggly solid line illustrates the density variation inside the plume, and four dashed lines represent plasma density contour.

sition, plume density variation and ring current proton properties are considered in section 4. In section 5, we summarize our principal conclusions. Finally, the paper ends with a discussion.

\section{Plasma Model for Ray Tracing}

[5] Here we use ray racing to calculate the propagation paths of EMIC waves in a non uniform plasma, and to evaluate the path integrated gain. We use the HOTRAY code [Horne, 1989] which has been applied to electromagnetic and electrostatic waves, including EMIC waves in the Earth's magnetosphere [Horne and Thorne, 1993, 1994]. The code can trace any type of wave in a hot magnetized plasma with weak growth or damping, provided the wave remains linear. The ray tracing method assumes that gradients in the medium, notably the plasma density and magnetic field gradients, are small compared to the wavelength, and that the gradients remain continuous. The HOTRAY code checks this condition at every step along the raypath. The ray tracing equations [Horne, 1989, equations (1) and (2)] are integrated with respect to time to find the new position, and wave number at each time step. The hot plasma dispersion relation is solved at each step along the raypath keeping the frequency constant and calculating a complex wave vector $\mathbf{k}$. The imaginary part of $\mathbf{k}, \mathbf{k}_{i}$, is used to calculate the path integrated gain. Landau and up to 100 cyclotron harmonic resonances are used to solve the hot plasma dispersion relation. More details of the method can be found in [Horne, 1989; Horne and Thorne, 1993, 1994].

[6] To perform ray tracing in a heterogeneous magnetospheric plasma, an analytical model with a smoothly varying spatial distribution of magnetic field and density is required. Here a dipole magnetic field model is used. For simplicity, the density model is assumed to have no azimuthal variation in density to allow two-dimensional ray tracing in a meridian plane. The adopted density model represents the storm time magnetosphere by including a plasmapause and a high-density plume with a fine-scale density variation. The precise location of the plasmapause and plume can be adjusted to fit observations. A smooth model for cold electron density is constructed as follows.

[7] 1. The electron density model combines an equatorial density model for the plasmasphere and trough [Sheeley et al., 2001] and field-aligned density variation [Denton et al., 2006]. The plasmasphere (ps) and trough (tr) density, $n_{\mathrm{ps}}$ and $n_{\text {tr }}$, can be expressed as

$$
n_{p s}=1390(3 / L)^{4.83}\left(L R_{E} / r\right)^{\alpha_{p s}}
$$

$$
\begin{aligned}
n_{t r}= & 124(3 / L)^{4.0}+36(3 / L)^{3.5} \cos (\{\text { MLT } \\
& \left.\left.-\left[7.7(3 / L)^{2.0}+12\right]\right\} \pi / 12\right)\left(L R_{E} / r\right)^{\alpha_{t r}}
\end{aligned}
$$

where $L$ is the $\mathrm{L}$ shell parameter, $R_{E}$ the Earth radii, $r$ the distance away from the center of the Earth. MLT is nominally chosen to be 18 , but the results could equally apply to other MLTs, and the parameters controlling latitudinal variation in number density, $\alpha_{\mathrm{ps}}$ and $\alpha_{\mathrm{tr}}$ are taken to be 0.8 and 2.0, respectively, following Denton et al. [2006]. The plasmapause is established with an inner edge $L_{p p i}=3.5$ and width $0.1 R_{E}$ (the center of plasmapause $L_{p p}=$ 3.55), which corresponds to a moderate storm time condition $K_{p}=4.6$ [Carpenter and Anderson, 1992].

[8] 2. The storm time plume (pl) is established over the $\mathrm{L}$ range $L_{0}<L<L_{1}$, where $L_{0}$ and $L_{1}$ are locations for the plume inner and outer edges, with a density fluctuations given by

$$
n_{p l}=\left[P-B\left(1-\cos \left(2 \pi N \frac{L-L_{0}}{L_{1}-L_{0}}\right)\right)\right] n_{t r}
$$

$P$ is a constant factor by which the density peaks in the plume are enhanced relative to trough density, and $B$ and $N$ are parameters controlling the magnitude and the number of density fluctuations inside the plume.

[9] 3. The four regions of plasma density, $n_{p s}$ for plasmasphere $L<L_{p p}, n_{t r}$ for the inner trough $L_{p p}<L<L_{0}$ and for the outer trough $L>L_{1}$, and $n_{p l}$ for the plume $L_{0}<L<L_{1}$, are adjusted to obtain a smooth electron density (and density gradient) distribution over a meridian plane, using the transition function of form [Gallagher et al., 2000]

$$
Y\left(x ; x_{0}, \Delta_{x}\right)=0.5 \tanh \left(3.4534 \frac{x-x_{0}}{\Delta_{x}}\right)+0.5
$$



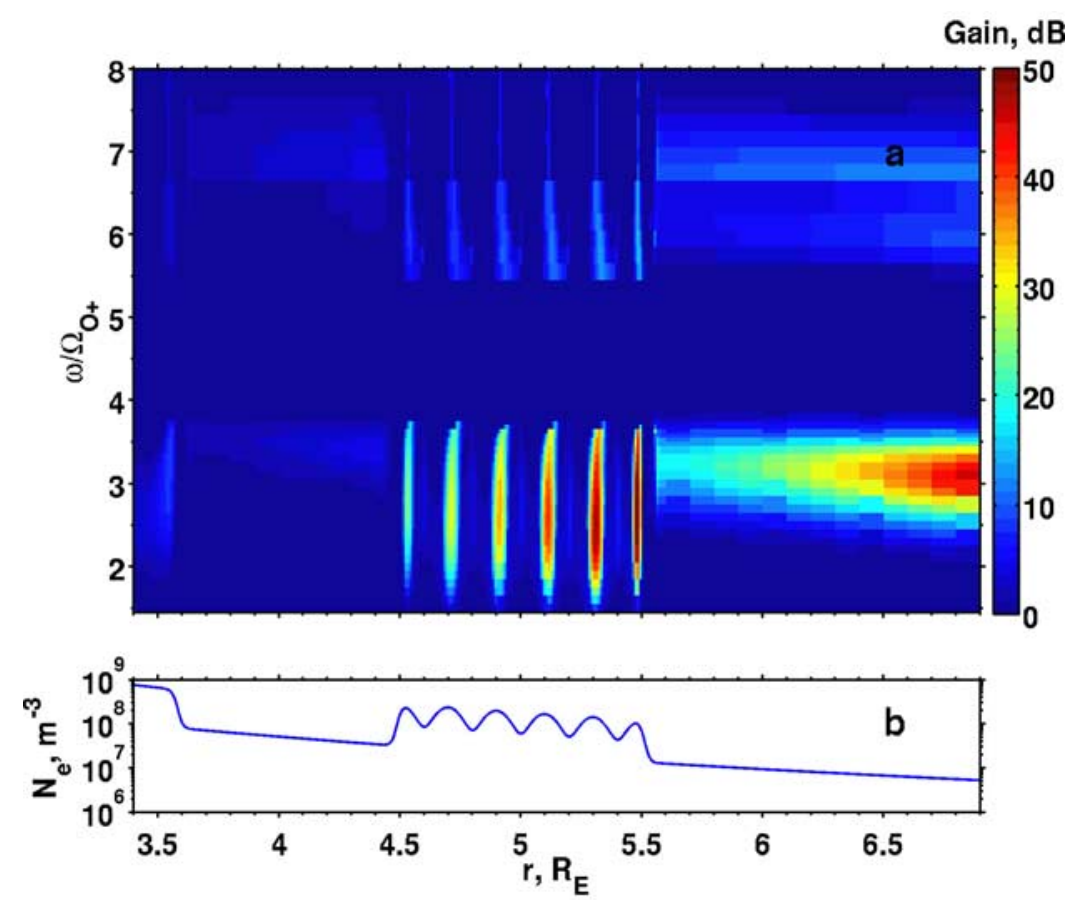

Figure 2. (a) Maximum wave gain as a function of normalized frequency and L shell for a bi-Maxwellian hot proton distribution (density $1 \mathrm{~cm}^{-3}, T_{\perp H^{+}}=50 \mathrm{keV}$ and $T_{\| H^{+}}=25 \mathrm{keV}$ ) and (b) the thermal plasma density model.

where $x_{0}$ is the midpoint for the transition and $\Delta_{x}$ is the range over which the transition occurs. Next we construct the electron density model as

$n_{e}=\left\{\begin{array}{l}n_{p s}\left(1-Y_{1}\right)+n_{t r} Y_{1} \text { if } L<\left(L_{p p}+L_{0}\right) / 2 \\ n_{t r}\left(1-Y_{2}\right)+n_{p l} Y_{2} \text { if }\left(L_{p p}+L_{0}\right) / 2<L<\left(L_{0}+L_{1}\right) / 2 \\ n_{p l}\left(1-Y_{3}\right)+n_{t r} Y_{3} \text { if } L>\left(L_{0}+L_{1}\right) / 2\end{array}\right.$

where $Y_{1}=Y\left(L ; L_{p p}, \Delta_{L_{p p}}\right), Y_{2}=Y\left(L ; L_{0}, \Delta_{L_{0}}\right)$ and $Y_{3}=$ $Y\left(L ; L_{1}, \Delta_{L_{1}}\right)$. A transition width of $0.1 R_{E}$ is assumed at the boundaries $L=L_{p p}, L=L_{0}$ and $L=L_{1}$, i.e., $\Delta_{L_{p p}}=$ $\Delta_{L_{0}}=\Delta_{L_{1}}=0.05$.

[10] Figure 2b shows the equatorial electron density as a function of $L$, with parameters $L_{0}=4.5, L_{1}=5.5, P=9$, $B=3$ and $N=5$. The value of $\mathrm{P}$ is selected to ensure that the ratio of electron plasma to gyrofrequency $\omega_{p e} /\left|\Omega_{e}\right|$ lies in the range from 10 to 20 inside the plume, consistent with measurement [e.g., Summers et al., 2008]. The upper and lower envelopes of the density inside the plume are $P n_{t r}$ and $(P-2 B) n_{t r}$, respectively. Thus the magnitude of density variation is about $2 B n_{t r}$ over one periodic length, which equals $\left(L_{1}-L_{0}\right) R_{E} / N=1 / 5 R_{E}$ in our simulation.

[11] A multi-ion $\mathrm{H}^{+}-\mathrm{He}^{+}-\mathrm{O}^{+}$magnetosphere plasma is adopted with uniform ion composition throughout the simulation region. Previous studies indicate heavy ion composition, $\eta_{\mathrm{He}^{+}}$and $\eta_{\mathrm{O}^{+}}$, in the range from $5 \%$ to $20 \%$ and from $1 \%$ to $10 \%$, respectively, but since the ion composition can vary substantially, $\eta_{\mathrm{He}^{+}}$and $\eta_{\mathrm{O}^{+}}$will be treated as free parameters to investigate the sensitivity on wave excitation and resulting electron cyclotron resonant energy.
[12] The thermal distribution for electron is assumed to be Maxwellian with an isotropic temperature of $10 \mathrm{eV}$ [Horne and Thorne, 1994]. The thermal ions have the same distribution with temperature $1 \mathrm{eV}$ for all $\mathrm{H}^{+}, \mathrm{He}^{+}$and $\mathrm{O}^{+}$ species. A hot ring current proton population is introduced as a source of free energy to drive the excitation of EMIC waves. The ring current proton distribution is assumed to be bi-Maxwellian with anisotropic temperature $T_{\perp H^{+}}>T_{\| H^{+}}$. For simplicity in the simulations the density of hot protons is fixed at $0.1 \mathrm{~cm}^{-3}$, independent of location, but the wave gain can be scaled up or down, on the basis of any measured profile of ring current proton density, since the wave gain is proportional to the number density of hot protons in the linear regime. Five free parameters remain to be specified in the model: $\eta_{\mathrm{He}^{+}}, \eta_{\mathrm{O}^{+}}, T_{\| H^{+}}, T_{\perp H^{+}}$(or anisotropy for hot $\mathrm{H}^{+}$ species $A_{H^{+}}=T_{\perp H^{+}} / T_{\| H^{+}}-1$ ), and $B$. A realistic spatial distribution of ring current proton density also need to be specified to obtain the radial wave gain profile.

\section{Raypath Integrated Gain}

[13] HOTRAY code [Horne, 1989] is used to trace the raypath and evaluate path integrated gain of $\mathrm{L}$ mode EMIC waves in the $\mathrm{He}^{+}$and $\mathrm{H}^{+}$bands using the plasma model described in section 2. Guided waves in the $\mathrm{He}^{+}$band occur with left hand polarization in the frequency range $\omega_{\text {cr } O^{+}}<\omega<$ $\Omega_{\mathrm{He}^{+}}$(mode 5 in the work by Horne and Thorne [1993]), where $\omega_{\mathrm{crO} \mathrm{O}^{+}}$is the $\mathrm{O}^{+}$crossover frequency and $\Omega_{\mathrm{He}^{+}}$the $\mathrm{He}^{+}$ gyrofrequency. Guided left-handed polarized waves in the $\mathrm{H}^{+}$band occur in the frequency range $\omega_{c r H e^{+}}<\omega<\Omega_{H^{+}}$ (mode 4 in the work by Horne and Thorne [1993]), where $\omega_{c r H e}$ is the $\mathrm{He}^{+}$crossover frequency and $\Omega_{H^{+}}$is the $\mathrm{H}^{+}$ gyrofrequency. All waves are initiated at the equator with a 

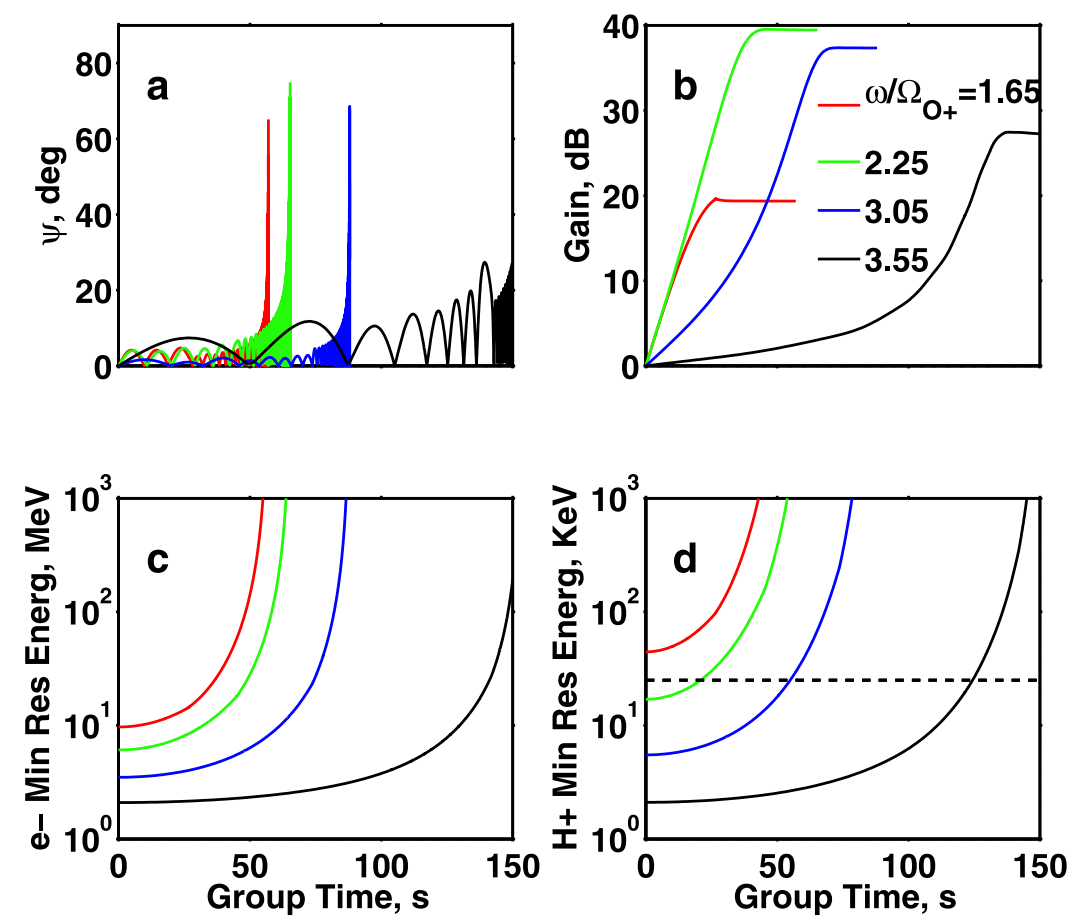

Figure 3. Ray tracing results for four different EMIC wave frequencies initiated inside the plume at $L=5.11$, where there is negative density gradient. (a) Wave normal angle versus group time, (b) wave gain, (c) minimum proton cyclotron resonant energy, and (d) minimum electron cyclotron resonant energy.

wave vector parallel to the ambient magnetic field. As the waves propagate, the polarization may become elliptical and even change to right handed below the crossover frequency, but the waves should remain on the same dispersion surface. The maximum wave gain along a raypath from the equator to the wave reflection location is obtained as a function of $L$ and wave frequency normalized to the equatorial $\mathrm{O}^{+}$gyrofrequency, $\omega / \Omega_{\mathrm{eqO}^{+}}$. An example of the radial profile of maximum gain is shown in Figure 2a using the electron density profile shown in Figure $2 \mathrm{~b}$ with $P=9, B=3, N=5$, ion composition $\eta_{\mathrm{He}^{+}}=10 \%$ and $\eta_{\mathrm{O}^{+}}=1 \%$, for a hot proton temperature $T_{\perp H^{+}}=50 \mathrm{keV}$ and $T_{\| H^{+}}=25 \mathrm{keV}$ and density $1.0 \mathrm{~cm}^{-3}$. The maximum gain shown in Figure $2 \mathrm{~b}$ would also be identical to the wave gain at the equator for waves which are traced backward along the raypath. The backward ray tracing can be done through the following two steps. First, waves are traced forward from the equator (with initial zero wave normal angle) to high latitude until they encountered a region where they are heavily damped. Then we reverse the $\mathbf{k}$ vector and the direction of ray tracing so that the waves propagate backward along the same raypaths until they cross the equator. In this way, any wave growth that takes place as the waves propagate toward high latitude should be identical to those waves propagating toward the equator, which should cross the equator with zero wave normal angle. Waves which experience strong wave gain on such a backward path are capable of resonating at the equator with the minimum energy electrons along the raypath. The wave gain can be scaled up or down to the measured density of ring current ions. Here, we arbitrarily normalize the gain to a hot proton density of $1 \mathrm{~cm}^{-3}$, which is comparable to the ring current proton density observed near $L \sim 5$ during storm time conditions [e.g., Frank, 1967].
[14] As noted earlier by Thorne and Horne [1997], moderate wave gain in the $\mathrm{He}^{+}$band occurs at the inner edge of the plasmapause, because of the strong negative density gradient. Such density gradients tend to refract the waves inward against the outward refraction caused by magnetic curvature: this ensures that the wave normal angle remains small over a significant raypath length, thus maximizing the wave gain. The gain in Figure $2 b$ is obtained by assuming $1 \mathrm{~cm}^{-3}$ hot proton density, which is probably an underestimate for storm time conditions. Consequently, gain near the plasmapause should be substantially larger when a more realistic profile of ring current density during storm time condition is adopted.

[15] For $N_{h o t}=1 \mathrm{~cm}^{-3}$, strongest gain in the $\mathrm{He}^{+}$band is confined to the outer trough region at large $\mathrm{L}$, and the extended region centered near the negative density gradients inside the plume. For the adopted parameters, wave gain in the $\mathrm{He}^{+}$band is substantially greater than the gain in the $\mathrm{H}^{+}$ band throughout the simulation domain. The increased wave gain in the low-density trough at larger L can be explained by the combined effect of the increase in the ratio $\omega_{p e} /\left|\Omega_{e}\right|$, which lowers the proton resonant energy, and the increase in the ratio of the number of resonant proton to the local plasma density [Kennel and Petschek, 1966], both of which tend to enhance the wave growth rate. Increasing $\omega_{p e} /\left|\Omega_{e}\right|$ also reduces the peak normalized frequency, because the lower-frequency waves can resonate with the majority of hot proton at the adopted temperature. The unstable wave frequency band in the outer trough is confined to the range from $2.5 \Omega_{O^{+}}$to $3.5 \Omega_{O^{+}}$.

[16] The gain for both the $\mathrm{He}^{+}$and $\mathrm{H}^{+}$bands inside the plume is strongly modulated by the density variation. Enhanced wave gain for both $\mathrm{He}^{+}$and $\mathrm{H}^{+}$band occurs over 

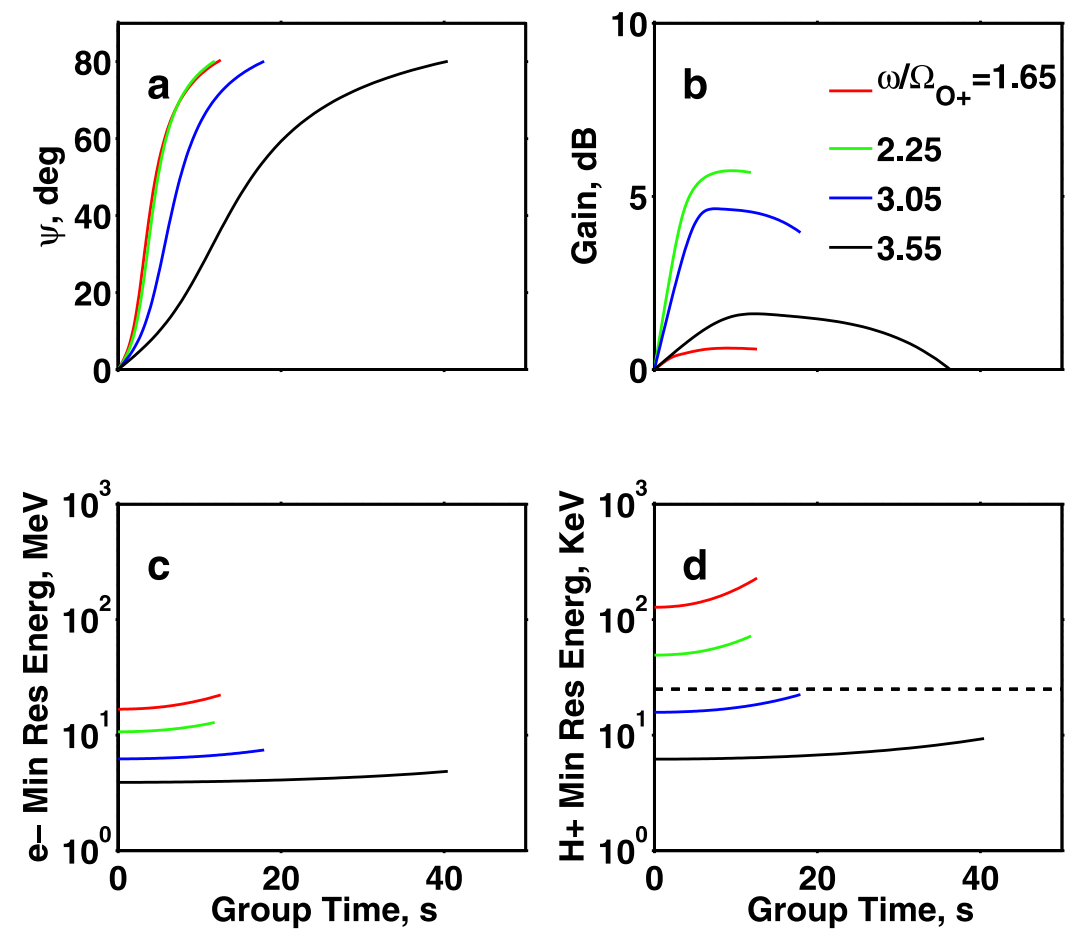

Figure 4. The same as Figure 3 except for ray tracing results at one of the density minimums inside the plume, $L=5.20$.

six separate narrow regions extending from just inside the density peaks to the negative density gradient side of the density peaks. The width of the former side is relatively narrow, compared to the latter. The location where the $\mathrm{He}^{+}$ band waves obtain peak gain for each of the six regions is slightly outside the corresponding density peak in regions of modest negative density gradient. The band of unstable wave frequency can extend from below $2 \Omega_{O^{+}}$to about $3.7 \Omega_{O^{+}}$, which is broader than the unstable wave band in the outer trough region. Waves excited at the frequencies above $3.5 \Omega_{O^{+}}$, which are able to resonate with relativistic electron of relatively lower energy, is also stronger near the density peak in the plume than that in the density minima. However, waves with frequencies very close to the $\mathrm{He}^{+}$ gyrofrequency $\left(>3.7 \Omega_{O^{+}}\right.$) are subject to severe damping because of cyclotron resonant absorption by thermal $\mathrm{He}^{+}$. The location of peak wave gain is due to a combination of wave refraction by negative density gradient, which keeps the waves more field aligned, and the heightened density with high value of $\omega_{p e} /\left|\Omega_{e}\right|$, which lowers ion resonant energy. Smaller wave gain occurs just inside the peak density where the density gradient is positive but small, primarily because of the heighten density and thus lower proton resonant energy.

[17] An interesting guiding phenomenon occurs for the waves starting at locations of negative density gradient. Both the raypath and wave normal angle of these waves exhibit oscillations around the magnetic fields. Figure 3 shows an example of propagation characteristics along the raypath of four waves with different frequencies starting slightly outside a density peak $(L=5.10)$ in the plume region at $L=5.11$. All the waves are initially guided along the magnetic field line, maintain small wave normal angles $\left(\leq 10^{\circ}\right)$ over a larger part of the raypath, and thus obtain larger gain. The behavior of the wave normal angle is a result of the competition between refraction due to plasma density gradients and magnetic field gradients and curvature: the negative (positive) radial density gradient tends to drive the wave vector inward (outward) while refraction by the dipole magnetic field gradients and curvature, which is enhanced at lower $L$, always tends to refract wave vector outward. The oscillating wave normal angle is caused by the balance between refraction by the negative density gradient and by the effect of the magnetic field, especially for the wave with $\omega=3.05 \Omega_{\text {eq }} O^{+}$(blue line) whose normal angle is less than $2^{\circ}$ over the first $70 \mathrm{~s}$. Initially all these waves tend to migrate toward smaller L shell because of stronger inward refraction associated with the steep negative density gradient. These waves are pulled back and toward the other side of the density peaks, where the magnetic refraction again dominates. These waves then migrate back to larger $L$. Rapid oscillations in both wave vector and wave position vector along the magnetic field line eventually occurs at the density peak location $(L=5.1)$, which is associated with ducting processes occurring in the density peak region.

[18] As waves propagate toward high latitude, the cyclotron resonant energy for both electrons and protons increases rapidly, because of the combination of lower $\omega / \Omega_{O^{+}}$at higher latitude and lower $\omega_{p e} / \Omega_{e}$. Waves with $\omega=2.25 \Omega_{O^{+}}$have relatively larger gain because the wave remains in cyclotron resonance over a longer time period with the major portion of the hot protons (with energy $25 \mathrm{keV}$ ). The waves are able to resonate with relativistic electron with several $\mathrm{MeV}$ parallel energy near the equator, but the resonant energy increases rapidly as waves propagate toward high latitudes. Excited wave fre- 

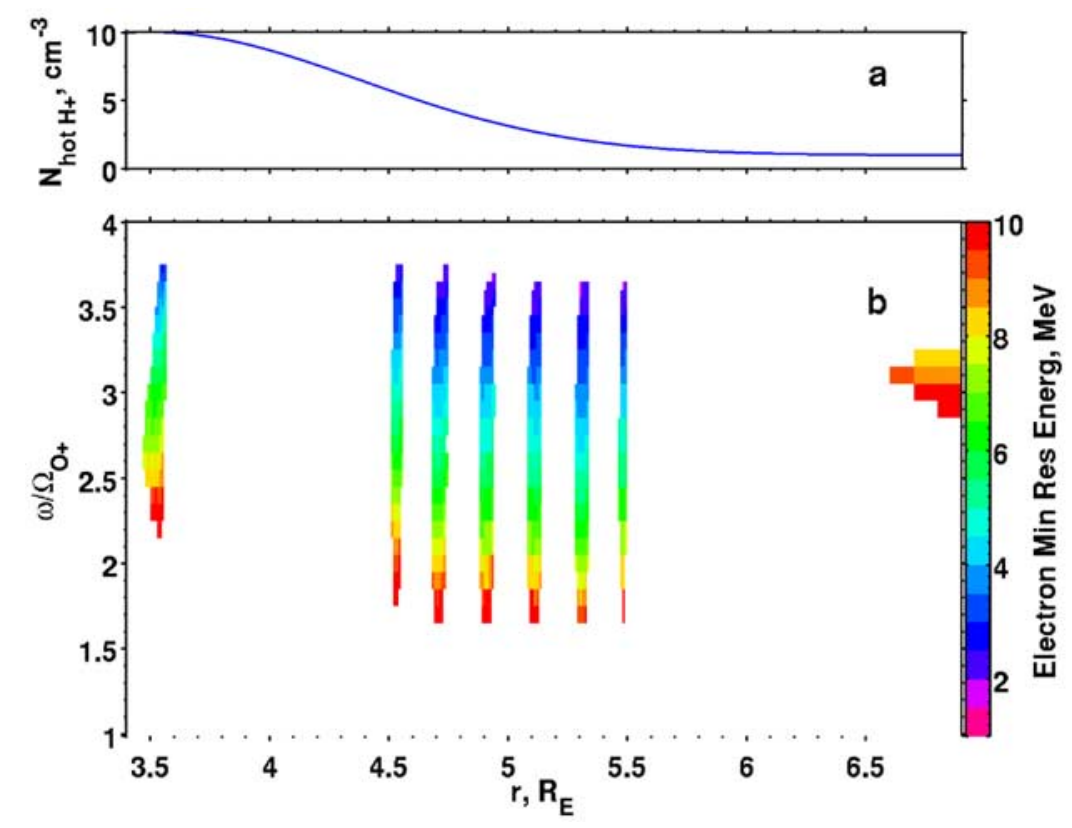

Figure 5. (a) Ring current proton density profile adopted to represent storm time profile. (b) Minimum electron resonant energy with wave gain greater than $40 \mathrm{~dB}$, assuming ring current proton density profile in Figure 5a. White area denotes the regime where wave gain is below $40 \mathrm{~dB}$.

quencies closer to $\Omega_{\mathrm{He}^{+}}$result in lower minimum electron resonant energy, because of the lower wave phase velocity.

[19] Examples of the propagation characteristics of waves starting at one of density minimum region $(L=5.2)$ are shown in Figure 4. In this case, the density gradient has little effect on the wave normal, and magnetic curvature and gradients cause the wave to be refracted rapidly to highly oblique wave normal angle. As a result, the integrated wave gain is significantly suppressed to generally less than $5 \mathrm{~dB}$, which is an order of magnitude smaller than wave gain near the density peaks.

[20] The ring current density is not uniform, but tends to maximize near the plasmapause [e.g., Frank, 1967; Thorne and Horne, 1997]. A realistic ring current density distribution is required to simulate the spatial location of excited EMIC waves. Under the conditions when wave growth rate is small enough to ignore nonlinear effect, and that the ring current density itself is small enough that the wave dispersion relation is primarily determined by the thermal species, one may scale the results shown in Figure 2 to any specified radial profile of ring current density. To simulate a realistic ring current density profile during storm conditions, we adopt a Gaussian distribution over $\mathrm{L}$, which has peak value $10 \mathrm{~cm}^{-3}$ at plasmapause location $L=3.5$, and $2.5 \mathrm{~cm}^{-3}$ at $L=5.0$, corresponding to the middle of the plume, $1 \mathrm{~cm}^{-3}$ at $L=7.0$, as shown in Figure 5a.

[21] We adopt the criterion that at least $40 \mathrm{~dB}$ of wave gain is required to allow waves to grow to observable levels from the background noise. The spatial regime and normalized frequency where one would anticipate significant wave excitation is indicated by the colored areas in Figure 5. The color itself denotes the minimum electron resonant energy with these excited waves. For the adopted parameters, no waves in the $\mathrm{H}^{+}$band obtain gain greater than $40 \mathrm{~dB}$. Excited waves in the $\mathrm{He}^{+}$band are confined to just inside the plasmapause, narrow regions near each density peak inside the plume and $L>6.5$ in outer trough. The broadest spectrum of waves $1.6 \Omega_{O^{+}} \leq \omega \leq 3.7 \Omega_{O^{+}}$is found inside the plume, the second broadest $2.2 \Omega_{O^{+}} \leq \omega \leq 3.75 \Omega_{O^{+}}$just inside the plasmapause, and the narrowest spectrum $\left(2.8 \Omega_{O^{+}} \leq \omega \leq 3.25 \Omega_{O^{+}}\right)$in the low-density outer trough region. Waves excited near the plasmapause are only able to resonate with electrons above $3 \mathrm{MeV}$. The minimum electron resonant energy with EMIC waves inside the plume is generally above $2 \mathrm{MeV}$, while EMIC waves excited in the outer trough can only resonant with electrons above at least $8 \mathrm{MeV}$.

\section{Sensitivity of Results to Model Parameters}

[22] The results described above were obtained with a set of five nominal but realistic free parameters: $\eta_{\mathrm{He}^{+}}=10 \%$, $\eta_{O^{+}}=1 \%, T_{\| H^{+}}=25 \mathrm{keV}, T_{\perp H^{+}}=50 \mathrm{keV}$, and $B=3$. To assess the robustness of the general features of our simulation of EMIC wave excitation, a sensitivity study is performed on each of these five free parameters, holding the other parameters constant and using the same ring current density profile shown in Figure 5a.

\subsection{Variable Ion Composition}

[23] The sensitivity of the EMIC wave gain and the corresponding minimum electron resonant energy to variation in ion composition is explored in Figure 6. Variations in the $\mathrm{O}^{+}$composition from $1 \%$ to $10 \%$ (holding the $\mathrm{He}^{+}$ composition constant) are considered in Figures $6 \mathrm{a}$ and $6 \mathrm{~b}$. When the $\mathrm{O}^{+}$composition is increased, the lower cutoff of excited $\mathrm{He}^{+}$band increases because of an increase in the $\mathrm{O}^{+}$ crossover frequency, while the upper cutoff is slightly reduced. An increase in $\mathrm{O}^{+}$composition also leads to a reduction in the wave gain and an increase in the electron minimum resonant energy over the entire excited $\mathrm{He}^{+}$band. In Figures $6 \mathrm{c}$ and $6 \mathrm{~d}$, the $\mathrm{O}^{+}$composition is kept constant 

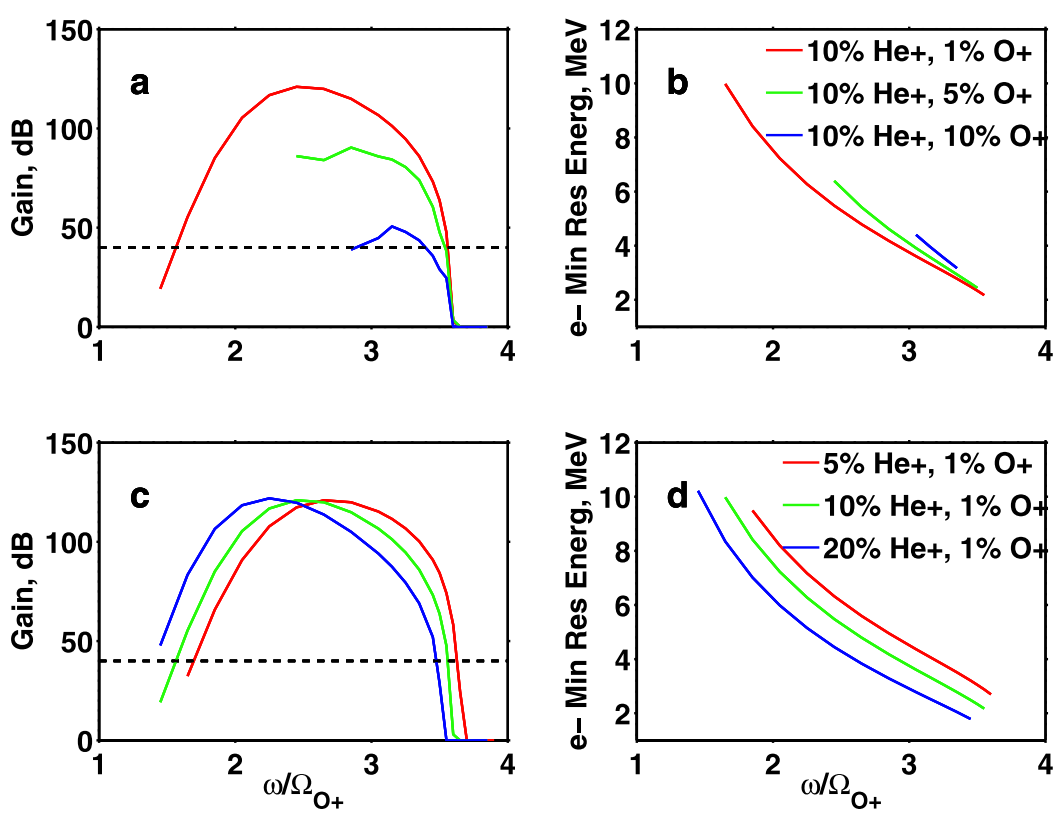

Figure 6. (a) Wave gain as a function of normalized frequency at one of the density peaks in the plume, $L=4.9$, under the conditions with different $\mathrm{O}^{+}$composition and the same $\mathrm{He}^{+}$composition. Horizontal dashed line denotes $40 \mathrm{~dB}$ gain. (b) The resulting minimum electron resonant energy with EMIC waves of gain exceeding $40 \mathrm{~dB}$ in Figure 6a. (c) The same as Figure 6a except for the conditions with different $\mathrm{He}^{+}$and the same $\mathrm{O}^{+}$composition. (d) The resulting minimum electron resonant energy from Figure 6c.

$(1 \%)$ and the $\mathrm{He}^{+}$composition is varied from $5 \%$ to $20 \%$. Larger $\mathrm{He}^{+}$composition results in a slight shift of excited wave band toward lower frequency with similar peak gain. Lowering of the upper cutoff frequency for excited waves is due to enhanced $\mathrm{He}^{+}$cyclotron resonance absorption at wave frequencies close to the $\mathrm{He}^{+}$gyrofrequency. Despite this, the minimum electron resonant energy is reduced to values below $2 \mathrm{MeV}$ for high $(20 \%) \mathrm{He}^{+}$composition, because of a lowering of the wave phase velocity.

\subsection{Plume Density Variation}

[24] The effect of varying the magnitude of the density variation is investigated in Figure 7 by varying the parameter $B$ in equation (3) from 3 to 0.25 . Increased density variation (and associated density gradients) enhances wave gain throughout the $\mathrm{He}^{+}$band, especially for wave frequencies above $2 \Omega_{O^{+}}$. However, waves with frequencies above $3.7 \Omega_{O^{+}}$are totally suppressed by the thermal $\mathrm{He}^{+}$cyclotron absorption, regardless of the magnitude of the density variation.

[25] Profiles of the wave gain and corresponding minimum electron resonant energy are shown in Figure 8 as a function of normalized wave frequency over one periodic length $(4.9 \leq L \leq 5.1)$ of density variation inside the plume, for different amplitude of density variation. Highest wave gain is found over a broader region on the negative density gradient side of the plume because of strong guiding of waves by the negative density gradients, which counteracts the effect of magnetic field gradients and curvature. Wave gain also occurs over a narrower region just before the density peak, primarily as a result of the heightened density, which lowers proton resonant energy and therefore increasing the growth rate. Wave gain inside the plume is smallest near the density minimum. As the overall density variation in the plume decreases (smaller $B$ ), the wave excitation region on the negative gradient side becomes broader, and the maximum gain is reduced and shifts toward lower frequency. Meanwhile the wave excitation region just inside the density peak slightly extends further inward. When the density variation is small, EMIC waves tends to grow to observable level throughout the whole region $(4.9 \leq L \leq$ 5.1), and the excited wave spectrum peaks at about $2 \Omega_{O^{+}}$,

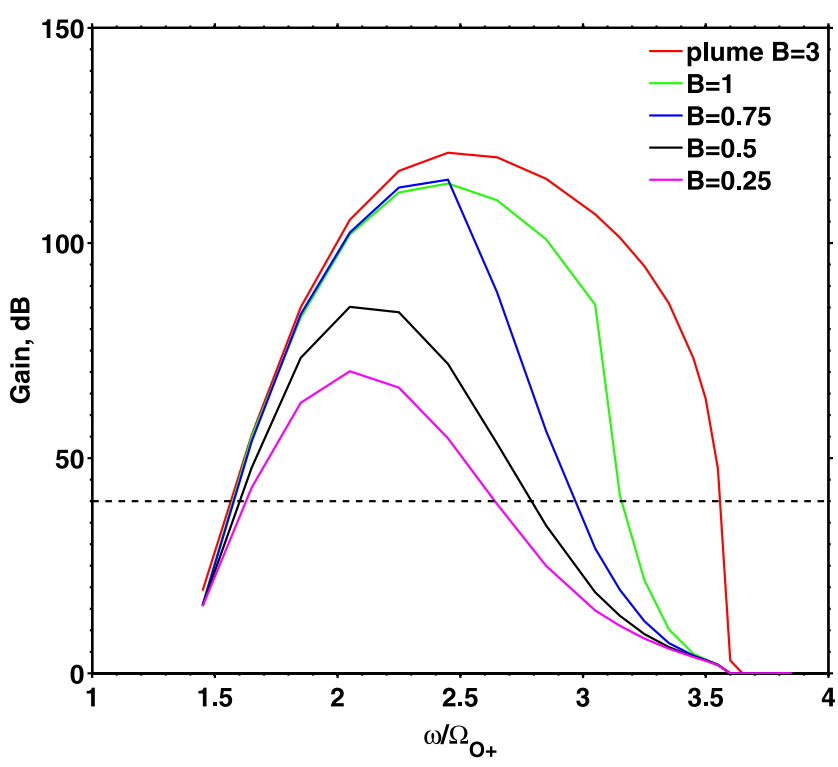

Figure 7. Wave gain as a function of normalized frequency at one of the plume density peaks, $L=4.90$, for different plume density variation parameters: $B=3, B=1$, $B=0.75, B=0.5$, and $B=0.25$. Horizontal dashed line denotes the $40 \mathrm{~dB}$ gain. 


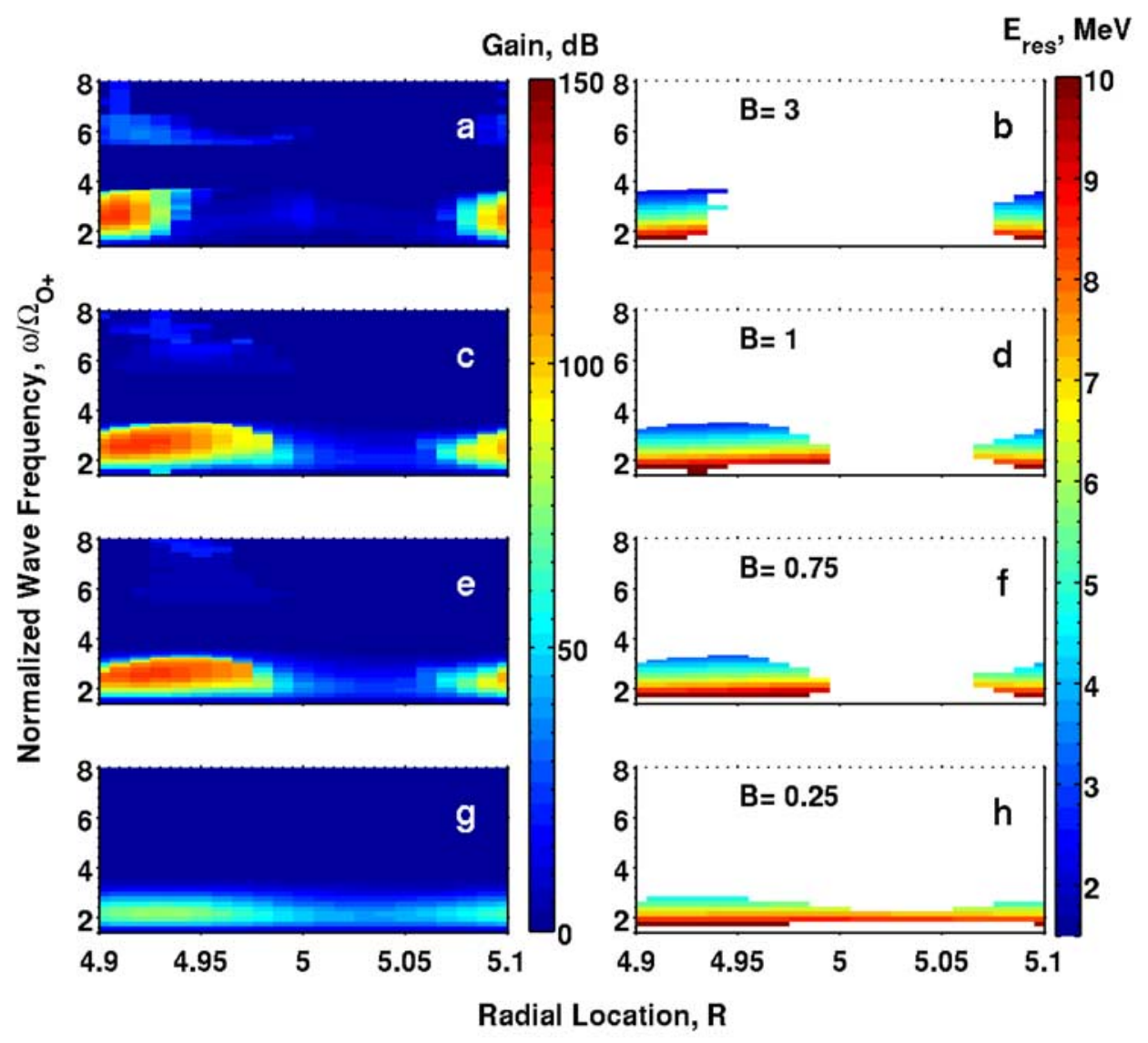

Figure 8. (a, c, e, and g) Wave gain distribution in the space of location and normalized wave frequency over one periodic length in the plume for different plume density variation parameters: $B=3, B=1$, $B=0.75$, and $B=0.25$ (Figures $8 \mathrm{a}, 8 \mathrm{c}, 8 \mathrm{e}$, and $8 \mathrm{~g}$, respectively). (b, $\mathrm{d}, \mathrm{f}$, and $\mathrm{h}$ ) Corresponding minimum electron resonant energy with waves of gain greater than $40 \mathrm{~dB}$. The minimum of the resonant energy over this $L$ range is found to be $1.95,2.59,3.14$, and $4.79 \mathrm{MeV}$ (Figures $8 \mathrm{~b}, 8 \mathrm{~d}, 8 \mathrm{f}$, and $8 \mathrm{~h}$, respectively).

as shown in Figure 8g. Consequently, EMIC waves excited inside a plume without significant density variation, are only able to resonate with electron above $5 \mathrm{MeV}$, compared to $2 \mathrm{MeV}$ when the density variation is larger.

\subsection{Variation of Hot Proton Temperature and Anisotropy}

[26] Figure 9 shows the wave gain in the $\mathrm{He}^{+}$band for different hot proton temperature. By decreasing the parallel temperature $T_{\| H^{+}}$of hot $\mathrm{H}^{+}$from $25 \mathrm{keV}$ to $10 \mathrm{keV}$ while keeping the anisotropy $A_{H^{+}}=1$, the wave gain in the $\mathrm{He}^{+}$ band is suppressed at lower frequencies. However, the excitation at high normalized wave frequencies is not significantly affected. Consequently, there will be little effect on the minimum energy for electron resonance. Wave gain decreases for all wave frequencies when the anisotropy decreases while keeping the parallel temperature the same.

[27] In order to simulate the enhancement of ring current anisotropy due to solar wind compression on the dayside magnetosphere at large $\mathrm{L}$, we perform a ray tracing in the trough at $\mathrm{L}=6.8$ for $T_{\| H^{+}}=10 \mathrm{keV}$ and allow the anisotropy to increase from 1 to 1.5 (Figure 10). Although increased anisotropy leads to enhanced wave gain at all frequencies, only waves in the $\mathrm{H}^{+}$band, with frequency just above the $\mathrm{He}^{+}$crossover frequency, are able to grow to observable level. This is consistent with observations of EMIC waves in outer magnetosphere [Anderson et al., 1990] and also with the earlier simulation of Horne and Thorne [1997].

\section{Summary of Principal Results}

[28] The principal conclusion in this study can be summarized as follows.

[29] 1. On the basis of a realistic model for the spatial distribution of thermal plasma and hot ring current ion, path integrated gain calculation with HOTRAY indicates three preferred regions of cyclotron resonant EMIC wave excitation: just inside the plasmapause, within high-density drainage plumes, and in the low-density trough at $L>6.5$.

[30] 2. For modest ring current anisotropy, the strongest wave amplification $(>40 \mathrm{~dB})$ occurs over a broad frequency band below the equatorial $\mathrm{He}^{+}$gyrofrequency, within highdensity structured drainage plumes.

[31] 3. Density structure in the plumes can guide waves, keeping the wave normal angle small, leading to an order of magnitude enhancement in net gain in regions where the radial density gradient is negative. 


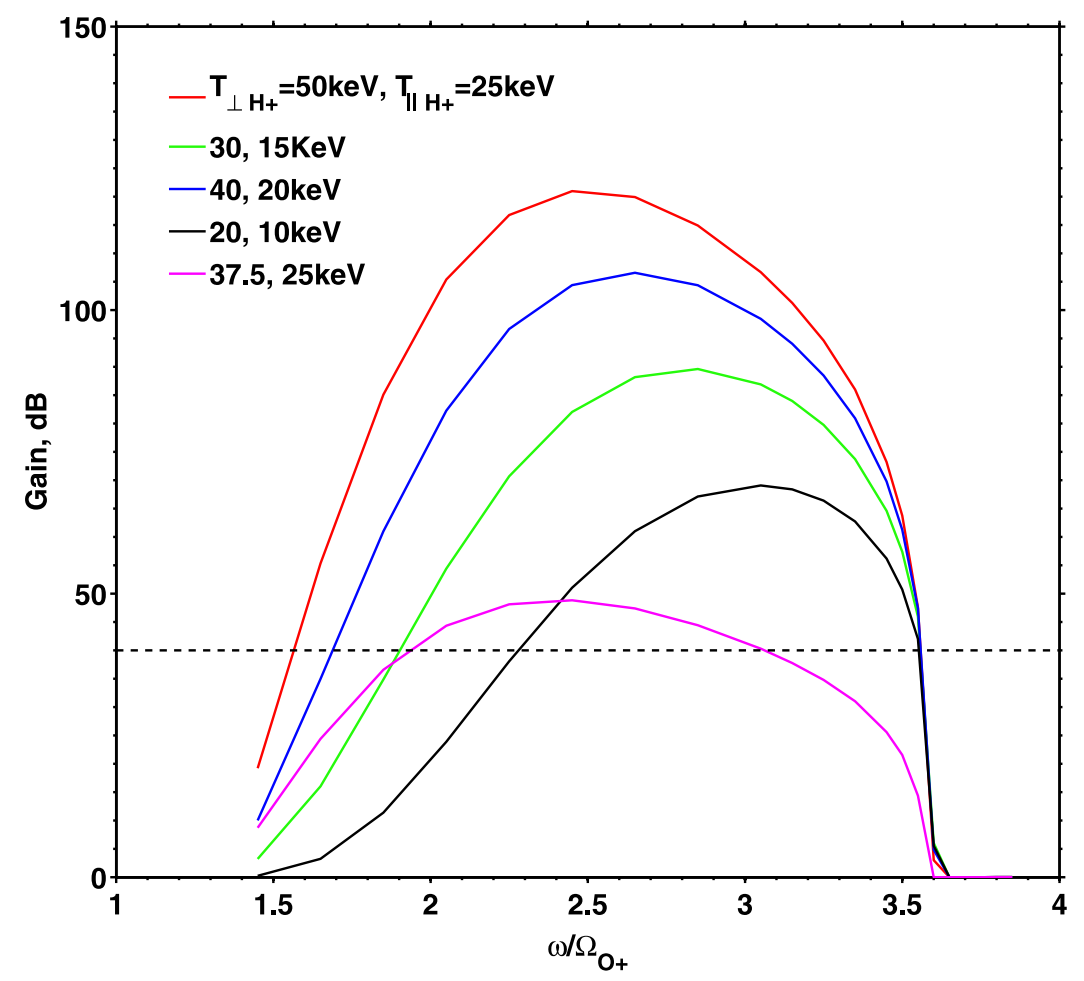

Figure 9. The same as Figure 7 except for different energetic proton temperatures.

[32] 4. Relativistic electrons can resonate with excited EMIC waves, causing rapid scattering loss. However, the minimum resonant electron energies tend to be several $\mathrm{MeV}$ for EMIC waves in plumes and near the plasmapause and
$>8 \mathrm{MeV}$ in the low-density trough. This result is relatively independent of adopted parameters in the simulation.

[33] 5. Observation of the $\mathrm{H}^{+}$band EMIC waves in the outer dayside magnetosphere can be explained by an

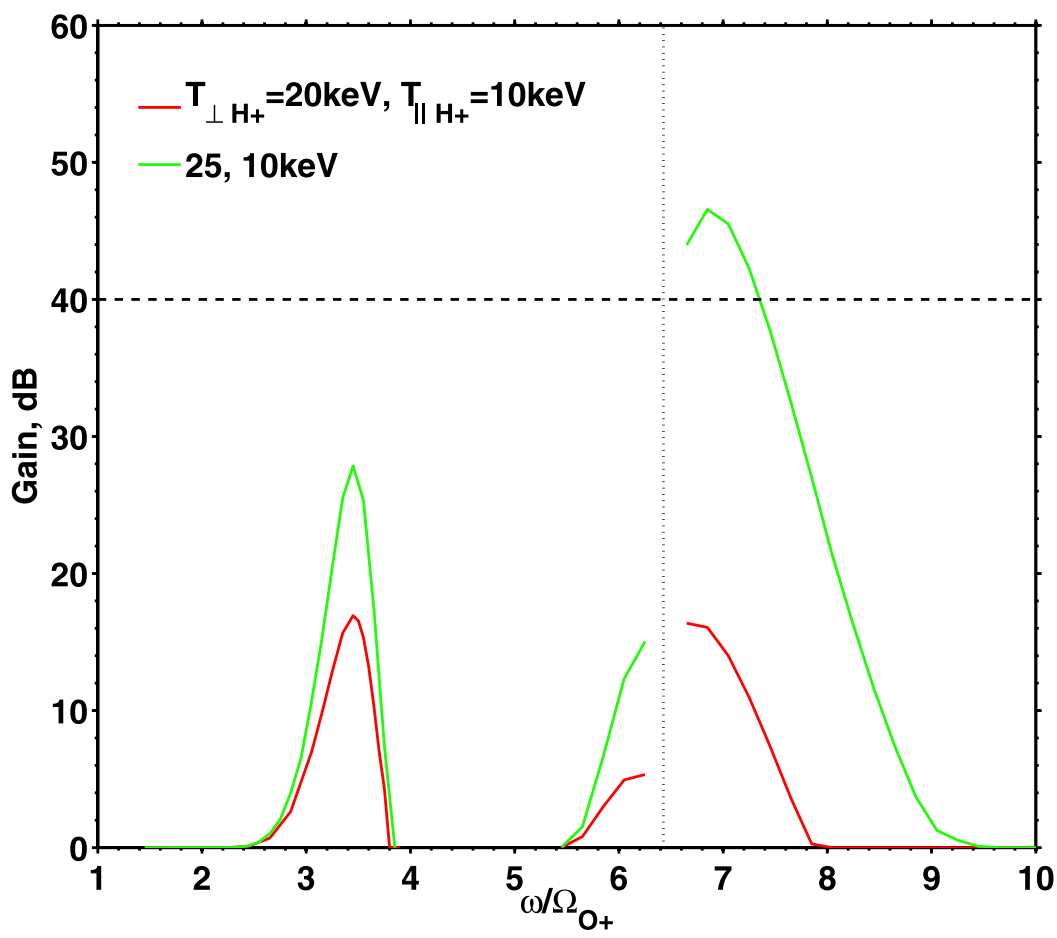

Figure 10. Wave spectrum at $L=6.8$ obtained with $T_{\| H^{+}}=10 \mathrm{keV}$ and two different $T_{\perp H^{+}}$values: $20 \mathrm{keV}$ (red line) and $25 \mathrm{keV}$ (green line). Vertical dotted line denotes the $\mathrm{He}^{+}$crossover frequency, $\omega_{c r H e^{+}}$. 
enhancement of ring current ion anisotropy $\left(A_{H^{+}}>1.5\right)$, possible as a result of solar wind compression.

\section{Discussion}

[34] EMIC waves are important in magnetospheric dynamics since they are able to cause heating of the thermal plasma [Thorne and Horne, 1992; Horne and Thorne, 1997], and pitch angle scattering and loss of both ring current ions [Cornwall et al., 1970] and relativistic electrons [e.g., Thorne and Kennel, 1971]. The interaction with relativistic electrons is particularly important since EMIC waves alone have the potential to cause strong diffusion scattering [Shprits et al., 2009], which could be the cause of sudden electron dropouts from the radiation belt [Millan et al., 2002]. However, the minimum energy for parasitic scattering of relativistic electrons is extremely dependent on both the frequency spectrum of the EMIC waves, and the composition and density of thermal plasma [e.g., Thorne et al., 2006; Li et al., 2007]. Specifically, the electron minimum energy is lower in locations where the phase speed of EMIC waves are reduced, which favors equatorial regions with high density and wave frequencies just below the $\mathrm{He}^{+}$ gyrofrequency [Summers and Thorne, 2003]. A quantitative assessment of the ability of EMIC waves to scatter relativistic electrons is complicated by the fact that, for a specified hot ion population, the most unstable excited wave frequencies tend to be lower (higher) in regions of higher (lower) density. EMIC waves also tend to have group velocities closely aligned with the magnetic field, which effectively prevents higher-frequency waves, excited in lower-density regions, from accessing high-density regions where the resonant electron energies are lower. Consequently, a selfconsistent simulation of wave excitation and associated electron scattering is required to better understand the potential of EMIC waves to cause scattering loss of relativistic electrons. Previous studies of such scattering have identified the global regions for EMIC excitation [e.g., Jordanova et al., 2008] and evaluated the rates of scattering from a specified EMIC wave spectrum [e.g., Li et al., 2007], but the earlier analyses of scattering rates have not been performed in a self-consistent manner. The analysis performed in the present study is the first to simulate the spectral properties of excited waves and directly utilize this information to evaluate electron resonant energies. The results confirm the basic conclusion of previous studies, which indicate that relativistic electrons are able to be scattered by EMIC waves excited during storm conditions. However, we find that such scattering is confined to energies typically greater than $2 \mathrm{MeV}$ in high-density regions (plasmapause and plumes) and greater than $8 \mathrm{MeV}$ in the low-density trough. This conclusion appears to be reasonably robust and is not very sensitive to precise values adopted for $\omega_{p e} / \Omega_{c e}$ or the ion composition.

[35] In future work we plan to test the predictions of our modeling of both the preferred regions of wave excitation and associated resonant electron scattering against available observation data, and incorporate the results into global modeling of the storm time dynamics of the ring current and radiation belt. Further improvements in the modeling should include the role of ring current $\mathrm{O}^{+}$ions, which tends to reduce EMIC instability in the $\mathrm{He}^{+}$frequency band [Thorne and Horne, 1997], and extension of ray tracing to three dimensions using more realistic model for the density structure in the inner magnetosphere.

[36] Acknowledgments. This research was supported by the NASA Heliosphere Theory grant NNX08A135G and NASA grant NNX08AF34G. [37] Amitava Bhattacharjee thanks Anatoly V. Streltsov and Jay Albert for their assistance in evaluating this paper.

\section{References}

Albert, J. M. (2003), Evaluation of quasi-linear diffusion coefficients for EMIC waves in a multispecies plasma, J. Geophys. Res., 108(A6), 1249 doi:10.1029/2002JA009792.

Anderson, B. J., K. Takahashi, R. E. Erlandson, and L. J. Zanetti (1990), Pc1 pulsations observed by AMPTE/CCE in the Earth's outer magnetosphere, Geophys. Res. Lett., 17, 1853-1856.

Carpenter, D. L., and R. R. Anderson (1992), An ISEE/Whistler model of equatorial electron density in the magnetosphere, J. Geophys. Res., 97(A2), 1097-1108.

Cornwall, J. M., F. V. Coroniti, and R. M. Thorne (1970), Turbulent loss of ring current protons, J. Geophys. Res., 75(25), 4699-4709, doi:10.1029/ JA075i025p04699.

Denton, R. E., K. Takahashi, I. A. Galkin, P. A. Nsumei, X. Huang, B. W. Reinisch, R. R. Anderson, M. K. Sleeper, and W. J. Hughes (2006), Distribution of density along magnetospheric field lines, J. Geophys. Res., 111, A04213, doi:10.1029/2005JA011414.

Erlandson, R. E., and A. J. Ukhorskiy (2001), Observations of electromagnetic ion cyclotron waves during geomagnetic storms: Wave occurrence and pitch angle scattering, J. Geophys. Res., 106(A3), 3883-3895.

Frank, L. A. (1967), On the extraterrestrial ring current during geomagnetic storms, J. Geophys. Res., 72(15), 3753-3767.

Gallagher, D. L., P. D. Craven, and R. H. Comfort (2000), Global core plasma model, J. Geophys. Res., 105(A8), 18,819-18,833.

Goldstein, J., B. R. Sandel, M. F. Thomsen, M. Spasojević, and P. H. Reiff (2004), Simultaneous remote sensing and in situ observations of plasmaspheric drainage plumes, J. Geophys. Res., 109, A03202, doi:10.1029/ 2003JA010281.

Gomberoff, L., and R. Neira (1983), Convective growth rate of ion cyclotron waves in a $\mathrm{H}^{+}-\mathrm{He}^{+}$and $\mathrm{H}^{+}-\mathrm{He}^{+}-\mathrm{O}^{+}$plasma, J. Geophys. Res., 88(A3), 2170-2174.

Horne, R. B. (1989), Path-integrated growth of electrostatic waves: The generation of terrestrial myriametric radiation, J. Geophys. Res., 94(A7), 8895-8909.

Horne, R. B., and R. M. Thorne (1993), On the preferred source location for the convective amplification of ion cyclotron waves, J. Geophys. Res., 98(A6), 9233-9247.

Horne, R. B., and R. M. Thorne (1994), Convective instabilities of electromagnetic ion cyclotron waves in the outer magnetosphere, J. Geophys. Res., 99(A9), 17,259-17,273.

Horne, R. B., and R. M. Thorne (1997), Wave heating of $\mathrm{He}^{+}$by electromagnetic ion cyclotron waves in the magnetosphere: Heating near the $\mathrm{H}^{+}$$\mathrm{He}^{+}$bi-ion resonance frequency, J. Geophys. Res., 102(A6), 11,45711,471 .

Jordanova, V. K., J. Albert, and Y. Miyoshi (2008), Relativistic electron precipitation by EMIC waves from self-consistent global simulations, J. Geophys. Res., 113, A00A10, doi:10.1029/2008JA013239.

Kennel, C., and H. Petschek (1966), Limit on stably trapped particle fluxes, J. Geophys. Res., 71(1), 1-28.

Li, W., Y. Y. Shprits, and R. M. Thorne (2007), Dynamic evolution of energetic outer zone electrons due to wave-particle interactions during storms, J. Geophys. Res., 112, A10220, doi:10.1029/2007JA012368.

Meredith, N. P., R. M. Thorne, R. B. Horne, D. Summers, B. J. Fraser, and R. R. Anderson (2003), Statistical analysis of relativistic electron energies for cyclotron resonance with EMIC waves observed on CRRES, J. Geophys. Res., 108(A6), 1250, doi:10.1029/2002JA009700.

Millan, R. M., R. P. Lin, D. M. Smith, K. R. Lorentzen, and M. P. McCarthy (2002), X-ray observations of $\mathrm{MeV}$ electron precipitation with a balloon-borne germanium spectrometer, Geophys. Res. Lett., 29(24), 2194, doi:10.1029/2002GL015922.

Sheeley, B. W., M. B. Moldwin, H. K. Rassoul, and R. R. Anderson (2001), An empirical plasmasphere and trough density model: CRRES observations, J. Geophys. Res., 106(A11), 25,631-25,641.

Shprits, Y. Y., L. Chen, and R. M. Thorne (2009), Simulations of pitch angle scattering of relativistic electrons with MLT-dependent diffusion coefficients, J. Geophys. Res., 114, A03219, doi:10.1029/2008JA013695. Spasojević, M., J. Goldstein, D. L. Carpenter, U. S. Inan, B. R. Sandel, M. B. Moldwin, and B. W. Reinisch (2003), Global response of the 
plasmasphere to a geomagnetic disturbance, J. Geophys. Res., 108(A9), 1340, doi:10.1029/2003JA009987.

Spasojević, M., H. U. Frey, M. F. Thomsen, S. A. Fuselier, S. P. Gary, B. R. Sandel, and U. S. Inan (2004), The link between a detached subauroral proton arc and a plasmaspheric plume, Geophys. Res. Lett., 31, L04803, doi:10.1029/2003GL018389.

Summers, D., and R. M. Thorne (2003), Relativistic electron pitch-angle scattering by electromagnetic ion cyclotron waves during geomagnetic storms, J. Geophys. Res., 108(A4), 1143, doi:10.1029/2002JA009489.

Summers, D., B. Ni, N. P. Meredith, R. B. Horne, R. M. Thorne, M. B. Moldwin, and R. R. Anderson (2008), Electron scattering by whistlermode ELF hiss in plasmaspheric plumes, J. Geophys. Res., 113, A04219, doi:10.1029/2007JA012678.

Thorne, R. M., and R. B. Horne (1992), The contribution of ion-cyclotron waves to electron heating and SAR-arc excitation near the storm-time plasmapause, Geophys. Res. Lett., 19, 417-420, doi:10.1029/ 92GL00089.

Thorne, R.M., and R.B. Horne (1997), Modulation of electromagnetic ion cyclotron instability due to interaction with ring current $\mathrm{O}^{+}$during magnetic storms, J. Geophys. Res., 102(A7), 14,155-14,163.
Thorne, R. M., and C. F. Kennel (1971), Relativistic electron precipitation during magnetic storm main phase, J. Geophys. Res., 76(19), 44464453 .

Thorne, R. M., R. B. Horne, V. K. Jordanova, J. Bortnik, and S. Glauert (2006), Interaction of EMIC waves with thermal plasma and radiation belt particles, in Magnetospheric ULF Waves: Synthesis and New Directions, Geophys. Monogr. Ser., vol. 169, edited by K. Takahashi et al., pp. 213-224, AGU, Washington, D. C.

Young, D. T., S. Perraut, A. Roux, C. de Villedary, R. Gendrin, A. Korth, G. Kremser, and D. Jones (1981), Wave-particle interactions near $\Omega_{\mathrm{He}^{+}}$observed on GEOS 1 and 2: 1. Propagation of ion cyclotron waves in $\mathrm{He}^{+}$-rich plasma, J. Geophys. Res., 86(A8), 6755-6772.

L. Chen and R. M. Thorne, Department of Atmospheric and Oceanic Sciences, University of California, Los Angeles, CA 90024, USA. (clj@atmos.ucla.edu)

R. B. Horne, British Antarctic Survey, Natural Environment Research Council, Cambridge CB3 0ET, UK. 